\title{
COMPARAÇÃO ENTRE CARGAS MEDIDAS E PREVISTA VIA FEM (FINITE ELEMENT METHOD) EM PROCESSO DE TREFILAÇÃO
}

\author{
Vanessa Moura de Souza ' \\ Tomaz Fantin de Souza ${ }^{2}$ \\ Mário Wolfart Júnior ${ }^{3}$ \\ Alexandre da Silva Rocha ${ }^{4}$
}

\section{Resumo}

O processo de trefilação de barras, arames e tubos é amplamente utilizado na indústria metal-mecânica para a fabricação de eixos automotivos, componentes mecânicos, cabos de aço, trilhos de trem, pregos, parafusos e etc. O conhecimento e controle dos parâmetros de processo como coeficiente de atrito, força de trefilação, tensões e deformações contribuem para uma melhor qualidade do produto e na redução do consumo energético e perdas desnecessárias. Desta forma, este trabalho objetiva analisar e medir a força necessária para trefilar uma redução em área do processo de fabricação de arames de aço de baixo teor de carbono, através da modelagem e simulação computacional do processo de trefilação e comparação dos resultados de força numéricos com os resultados experimentais obtidos através da trefilação dos arames em uma máquina universal de ensaios adaptada para a realização do processo. Além disso, este artigo tem como propósito indicar o coeficiente de atrito de Coulomb $(\mu)$ do processo utilizando o valor da força de trefilação obtida nos experimentos e a equação teórica de Siebel.

Palavras-chave: Trefilação de arames; Simulações computacionais; Força de trefilação; Coeficiente de atrito.

\section{NUMERICAL AND EXPERIMENTAL ANALYSIS OF STRENGTH FOR WIRE DRAWING LOW CARBON STEEL}

\begin{abstract}
The process of wire drawing is widely used in the metal industry for the manufacture of automotive axles, mechanical components, steel cables for the fishing industry, railroad tracks, nails, screws, etc. The knowledge and control of process parameters such as the coefficient of friction, wire drawing strength, stress and strain can contribute to a better quality of the product and in achieving a cleaner process, which reduces energy consumption and unnecessary losses. This paper aims at analyzing and measuring the necessary strength to wire drawing an area reduction in the manufacturing process of low carbon steel wire, through an Emic ${ }^{\circledR}$ universal testing machine adapted to the process and the construction of a device to hold the draw die. In addition, this paper aims to indicate Coulomb's coefficient of friction $(\mu)$ for the process using the value of the wire drawing strength obtained during experiments using the theoretical equation of Siebel and the strain and residual stress obtained through computational simulation results.
\end{abstract}

Keywords: Wire drawing; Computational simulations; Wire drawing strength; Coefficient of friction.

\section{INTRODUÇÃO}

Trefilação é um processo de fabricação que ocorre através do alongamento de um material e consequente redução em área de seção transversal, obtido pela passagem forçada do material pela fieira, também chamada matriz cônica, mediante a aplicação de uma força de tração na ponta do material. A deformação ocorre à medida que o material atravessa a fieira tendo seu diâmetro reduzido [I-4].

Um parâmetro muito importante a ser considerado é o atrito entre o material trefilado e a fieira, ferramenta responsável pela redução de diâmetro do material. $\mathrm{O}$ atrito

'Laboratório de Transformação Mecânica - LdTM, Universidade Federal do Rio Grande do Sul - UFRGS, Porto Alegre, RS, Brasil.

E-mail: vanessamouradesouza@gmail.com

${ }^{2}$ Departamento da Engenharia Mecânica, Instituto Federal Sul Rio-Grandense - IFSul, Sapucaia do Sul, RS, Brasil.

${ }^{3}$ Departamento da Engenharia Mecânica, Instituto Federal Catarinense - IFC, Luzerna, SC, Brasil.

${ }^{4}$ Departamento da Metalurgia, Universidade Federal do Rio Grande do Sul - UFRGS, Porto Alegre, RS, Brasil.

2176-1523 (C) 2017 Associação Brasileira de Metalurgia, Materiais e Mineração. Publicado pela ABM. Este é um artigo de acesso aberto distribuído sob os termos da licença Creative Commons CC BY-NC-ND (Attribution-NonCommercial-NoDerivs) https://creativecommons.org/licenses/by-nc-nd/4.0/. 
influencia diretamente nos processos de fabricação, e surge nas interfaces onde existe movimento relativo entre o material e a ferramenta. É um fenômeno indesejável na maioria das vezes por exigir, com seu aumento, maior potência para efetuar a operação, além de influenciar no acabamento superficial da peça e na deformação do material alterando valores de força e a vida em desgaste das ferramentas [5]. Outro parâmetro básico em um processo de trefilação é a força necessária para 0 material atravessar a fieira pois esta diretamente relacionado com o percentual de redução do material; quanto maior é a redução, maior a força necessária [6].

Desde os anos 60, com o desenvolvimento do Método de Elementos Finitos (FEM) e a sua aplicação através de métodos computacionais, a simulação numérica é uma ferramenta que está sendo cada vez mais utilizada para o conhecimento e otimização dos processos de fabricação. A utilização da simulação numérica permite evitar gastos desnecessários e perdas, investimentos em protótipos e testes dispendiosos, bem como para melhorar a qualidade do produto acabado [7-9].

Neste trabalho são comparados os valores da força de trefilação necessária para fabricar arames de aço de baixo teor de carbono, para uma redução, medidos através de diferentes técnicas, tais como: elementos finitos, medições experimentais e equação de Siebel. Com este trabalho foi obtido um comparativo entre os diversos métodos de medição da força de trefilação, maior compreensão do processo e das propriedades mecânicas de arames trefilados.

\section{MATERIAIS E MÉTODOS}

\section{I Ensaios de Trefilação}

Foram realizados ensaios de trefilação para arames de 5,50 $\mathrm{mm}$ de diâmetro e $100 \mathrm{~mm}$ de comprimento para duas diferentes corridas de um aço de baixo teor de carbono identificado pelo fabricante como aço AISI 1006. Estes ensaios foram desenvolvidos em uma máquina universal de ensaios Emic $^{\circledR}$ com capacidade máxima de 100 kN, do Laboratório de Metalurgia do Instituto Federal Catarinense IFC, Câmpus Luzerna. Na Tabela I são apresentadas características técnicas da mesma.

Através dos ensaios de trefilação foi possível medir a força necessária para realizar o processo e, aplicando a

Tabela I. Características Técnicas da Máquina Universal de Ensaios Emic

\begin{tabular}{lc}
\hline \multicolumn{1}{c}{$\begin{array}{c}\text { Capacidade } \\
(\mathbf{k g f})\end{array}$} & 1000 \\
\hline Acionamento & Eletromecânico \\
Faixa de Velocidade $(\mathbf{m m} / \mathbf{m i n})$ & 0,02 a 500 \\
Faixa de Abertura $(\mathbf{m m})$ & 120 a 1370 \\
Peso aproximando $(\mathbf{k g})$ & 420 \\
\hline
\end{tabular}

Fonte: Manual da Emic. equação de Siebel, identificar o coeficiente de atrito de Coulomb $(\mu)$. O conhecimento do valor do coeficiente de atrito de Coulomb deste processo é de fundamental importância como dado inicial (input) para o software de simulações computacionais.

A máquina de ensaios foi utilizada no módulo de tração para imprimir a força necessária para que 0 arame atravessasse a fieira. Na parte inferior foi acoplada a ferramenta de trefilação em um dispositivo desenvolvido para tal fim. O croqui do dispositivo é apresentado na Figura I.

Este dispositivo foi usinado em aço AISI I020, possui dimensões de $71 \mathrm{~mm}$ de altura com uma reentrância de $42,5 \mathrm{~mm}$ de profundidade, comprimento total da fieira, onde a ferramenta de trefilação foi acoplada. $O$ dispositivo possui quatro furos com o mesmo diâmetro dos parafusos disponibilizados pelo fabricante $\left(\mathrm{Emic}^{\circledR}\right)$ e devidamente fixados à máquina de ensaios. Na Figura 2 podemos ver esse dispositivo acoplado à fieira.

$\mathrm{Na}$ Tabela 2 são apresentados os parâmetros utilizados para a realização dos ensaios de trefilação. Foram ensaiados três corpos de prova para cada uma das corridas do material objeto deste estudo. A força foi adquirida através do software Tesc ${ }^{\circledR}$ integrado à máquina de ensaios. $O$ valor do coeficiente de atrito foi obtido inserindo o valor da força de equações empíricas encontradas na literatura.

\subsection{Simulações Numéricas Computacionais}

Foi desenvolvido um modelo numérico computacional para reproduzir o experimento. As simulações foram realizadas no software Simufact.forming ${ }^{\circledR}$ do Grupo de Engenharia de Superfícies do Laboratório de Transformação Mecânica (GES-LdTM) da Universidade Federal do Rio Grande do Sul. Na Tabela 3 são apresentados os parâmetros das simulações numéricas computacionais realizadas para descrever o experimento.

Gerou-se um modelo numérico a partir do software Simufact.formingGP ${ }^{\circledR}$ para o estudo do estado de tensões residuais gerado pelo processo de trefilação do aço de baixo teor de carbono. Primeiramente, desenvolveu-se um modelo axissimétrico bidimensional, Figura 3, que permite
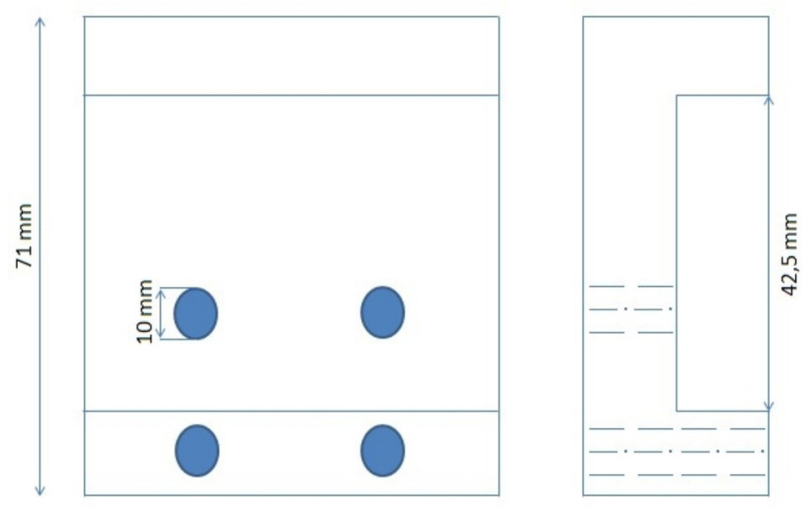

Figura I. Croqui do dispositivo de trefilação. 
que se simule uma fatia da barra, porém os resultados são extrapolados para o restante da geometria, o que permite reduzir o tempo de cálculo computacional. Neste modelo,

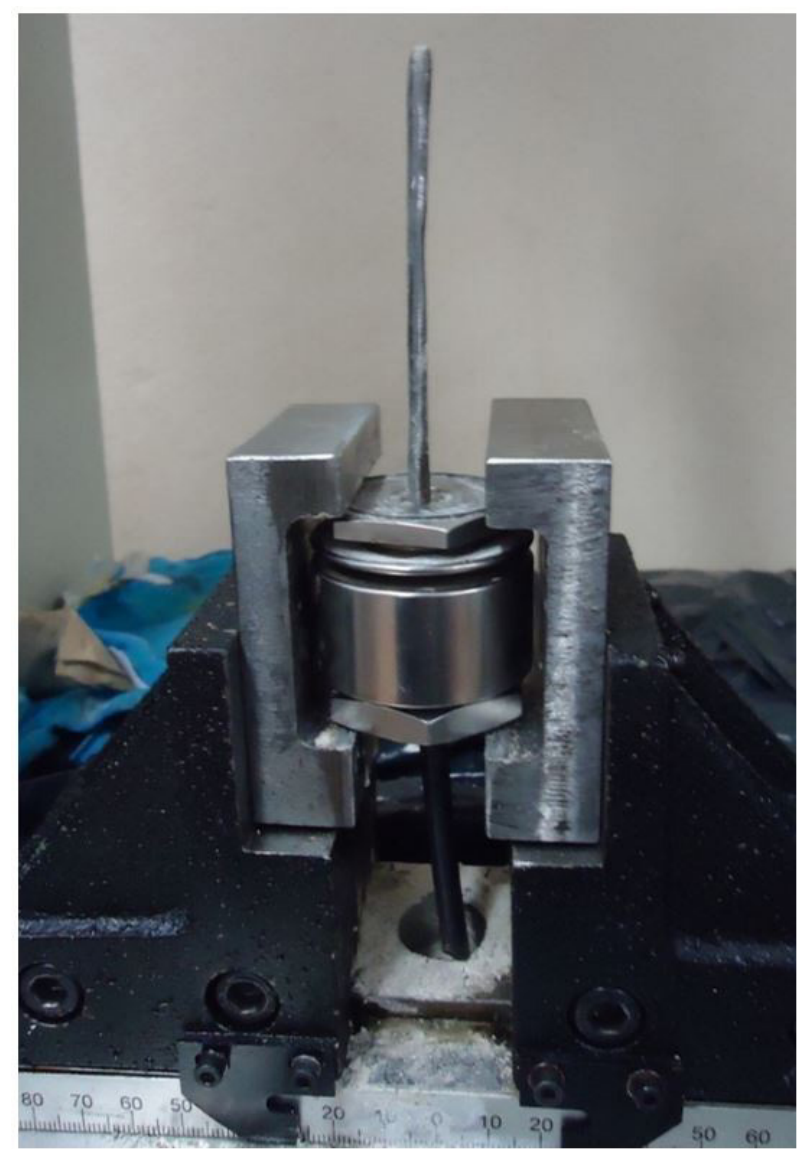

Figura 2. Dispositivo com a fieira acoplados na máquina de ensaios.

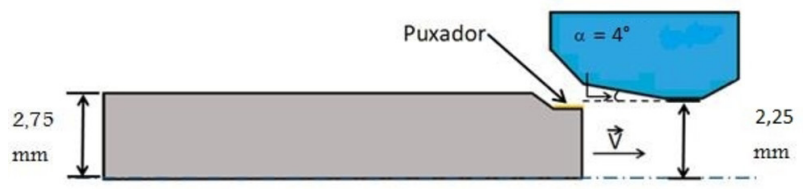

Figura 3. Modelo para trefilação.

Tabela 2. Parâmetros do material e fieira utilizados no ensaio

\begin{tabular}{lc}
\hline \multicolumn{1}{c}{ Tipo de ensaio } & Trefilação \\
\hline Equipamento & Máquina de Ensaio Universal Emic DL \\
& 10000 \\
Capacidade & $100000 \mathrm{~N}$ \\
Material & AISI I006 (aço de baixo teor de carbono) \\
Diâmetro de entrada & $5,5 \mathrm{~mm}$ \\
Diâmetro de saída & $4,5 \mathrm{~mm}$ \\
Lubrificantes & Sabão Indústrial de Trefila \\
Velocidade de trefilação & $2 \mathrm{~mm} / \mathrm{s}$ \\
Temperatura inicial & $20^{\circ} \mathrm{C}$ \\
Deformação & 0,414 \\
Ângulo de fieira $(2 \alpha)$ & $8^{\circ}$ \\
\hline
\end{tabular}

por considerações geométricas de simetria e a fim de diminuir o tempo de cálculo foi simulada metade do arame.

$\mathrm{O}$ arame modelado possui $100 \mathrm{~mm}$ de comprimento e $2,75 \mathrm{~mm}$ de diâmetro e a fieira tem comprimento total de $42,5 \mathrm{~mm}$.

Os valores das tensões residuais para as direções tangencial e axial após trefilação devem ser próximos na superfície, o que condiz com o resultado simulado [I0].

\section{RESULTADOS E DISCUSSÃO}

\section{I Força de Trefilação}

Na Tabela 4 são apresentados os dados utilizados para trefilação prática, enquanto na Tabela 5 são apresentados os resultados da força de trefilação dos experimentos. A tensão de escoamento inicial foi obtida do atlas da ASM para aços 1006, já a tensão de escoamento final foi obtida do software da Emic.

Foram realizados ensaios para três corpos de prova para cada uma das duas corridas do aço de baixo teor de carbono objeto deste estudo. Onde corrida I e II significam os testes executados nos dois rolos (carretel) de arame de aço de baixo teor de carbono fabricados em dias e momentos diferentes.

Para a corrida I, a força de trefilação variou de um valor mínimo de 5355,8 $\mathrm{N}$ até um valor máximo de $5462,8 \mathrm{~N}$ demonstrando uma diferença de $2 \%$ em valores percentuais. Já para a corrida II, a força de trefilação variou de um mínimo de 6167,6 N até um valor máximo de 6373, I N, apresentando uma diferença de $3 \%$ em valores percentuais.

A diferença nos resultados para a força de trefilação dos ensaios experimentais é considerada aceitável e demonstra uma boa capacidade da máquina em medir os valores de força. Esta diferença pode ser originada em limitações relacionadas a máquina ou do software de aquisição de dados; diferenças na lubrificação do processo realizado a seco; diferenças dimensionais, devido ao esmerilhamento da ponta do arame na etapa de apontamento do fio máquina e de inclusões e impurezas nos arames oriundos do processo de solidificação.

Tabela 3. Parâmetros das simulações numéricas computacionais

\begin{tabular}{|c|c|}
\hline Análises & $\begin{array}{l}\text { Mecânicas Axissimétricas } \\
\text { [Elementos Finitos] }\end{array}$ \\
\hline Número de elementos & $\begin{array}{c}500 \text { longitudinais } \times 5 \text { radias } \\
{[\text { Tipo QUAD] }}\end{array}$ \\
\hline Material & $\begin{array}{c}\text { Aço de baixo teor de carbono AISI } \\
1008 \text { [banco de dados] }\end{array}$ \\
\hline Velocidade de trefilação & $2 \mathrm{~mm} / \mathrm{s}$ \\
\hline Diâmetro inicial e final & $5,5 \mathrm{~mm} / 4,5 \mathrm{~mm}$ \\
\hline Temperatura inicial & $20^{\circ} \mathrm{C}$ \\
\hline Deformação verdadeira & $0,4 \mid 4$ \\
\hline Ângulos de fieira $(2 \alpha)$ & $8^{\circ}$ \\
\hline Módulo de Young & $210 \mathrm{GPa}$ \\
\hline
\end{tabular}


Tabela 4. Dados utilizados para trefilação prática experimental

\begin{tabular}{cccccc}
\hline Semiângulo Fieira & Área Inicial & Área Final & Deformação & $\begin{array}{c}\text { Tensão de } \\
\text { Escoamento Inicial }\end{array}$ & $\begin{array}{c}\text { Tensão de } \\
\text { Escoamento Final }\end{array}$ \\
\hline $\begin{array}{c}\alpha=0,0666 \mathrm{rad} \\
\text { ou 4 }\end{array}$ & $A_{0}=23,75 \mathrm{~mm}^{2}$ & $\mathrm{Al}=15,9 \mathrm{~mm}^{2}$ & $\varphi_{\mathrm{A}}=0,4$ & $k f_{0}=200 \mathrm{MPa}$ & $\mathrm{k}_{\mathrm{fl}}=450 \mathrm{MPa}$ \\
\hline
\end{tabular}

Tabela 5. Comparação entre os resultados de força experimentais

\begin{tabular}{cc}
\hline Aços baixo carbono & $\begin{array}{c}\text { Força de Trefilação Prática } \\
\text { (N) }\end{array}$ \\
\hline Corrida I & $5358,2 \mathrm{~N}$ \\
& $5355,8 \mathrm{~N}$ \\
Corrida II & $5462,8 \mathrm{~N}$ \\
& $6282,7 \mathrm{~N}$ \\
& $6373,1 \mathrm{~N}$ \\
$6167,6 \mathrm{~N}$ \\
\hline
\end{tabular}

Além disso, os arames não possuíam retilineidade igual, o que pode ter contribuído para a diferença encontrada entre as duas corridas.

\subsection{Atrito do Processo}

$\mathrm{Na}$ Tabela 6 são apresentados os resultados do coeficiente de atrito de Coulomb para a força de trefilação obtida através dos experimentos. Aplicando-se a Equação de Siebel para os valores de força medidos experimentalmente, pode-se encontrar um valor para o Coeficiente de atrito de Coulomb de 0, I para a corrida I e um valor de 0, I 2 para a corrida II do aço de baixo teor de carbono.

Este valor de coeficiente de atrito é condizente com a literatura pesquisada [8] que cita valores próximos a 0,1 para o processo de trefilação. As diferenças entre as duas corridas devem-se principalmente às diferenças no processo de solidificação do material. Duas diferentes corridas significam dois processos diferentes, concentração de impurezas, limites de escoamento e características diferentes, o que também justifica a diferença na força de trefilação medida experimentalmente entre as duas corridas.

\subsection{Simulações}

Nas Figuras 4 e 5 são apresentados o início da simulação e o modelo de elementos finitos desenvolvido para obtenção dos resultados de força, deformações e tensões residuais. É possível visualizar na Figura 4 a ferramenta e a barra (arame) no software de simulação já na Figura 5 podemos verificar o início do processo onde é aplicada a força de trefilação, ou seja, o esforço necessário para a barra passar pela fieira e deformar-se conforme a matriz.

\subsection{Força de Trefilação}

$\mathrm{Na}$ Tabela 7 são apresentados os resultados para a força de trefilação medida experimentalmente e simulada através de elementos finitos. Através das simulações
Tabela 6. Resultados do coeficiente de atrito de Coulomb

\begin{tabular}{cc}
\hline Aços baixo carbono & $\begin{array}{c}\text { Coeficiente de Atrito de } \\
\text { Coulomb }(\boldsymbol{\mu})\end{array}$ \\
\hline Corrida I & $0,1^{ \pm 0,2}$ \\
Corrida II & $0,12^{ \pm 0,2}$ \\
\hline
\end{tabular}

Tabela 7. Resultados para a força de trefilação obtidas por elementos finitos

\begin{tabular}{ccc}
\hline Simulação & Corrida I & Corrida II \\
\hline $8376 \mathrm{~N}$ & $5391 \mathrm{~N} \pm 866,32$ & $6275 \mathrm{~N} \pm 866,32$ \\
\hline
\end{tabular}

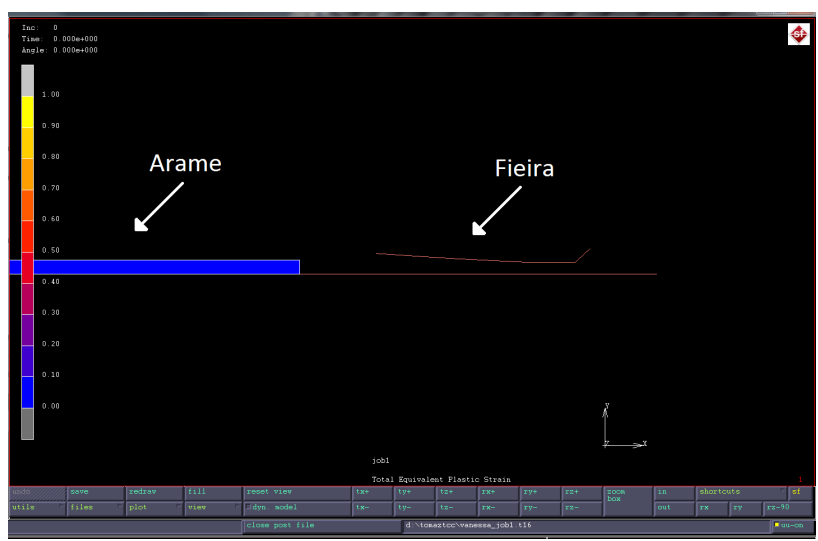

Figura 4. Início do processo de trefilação simulada.

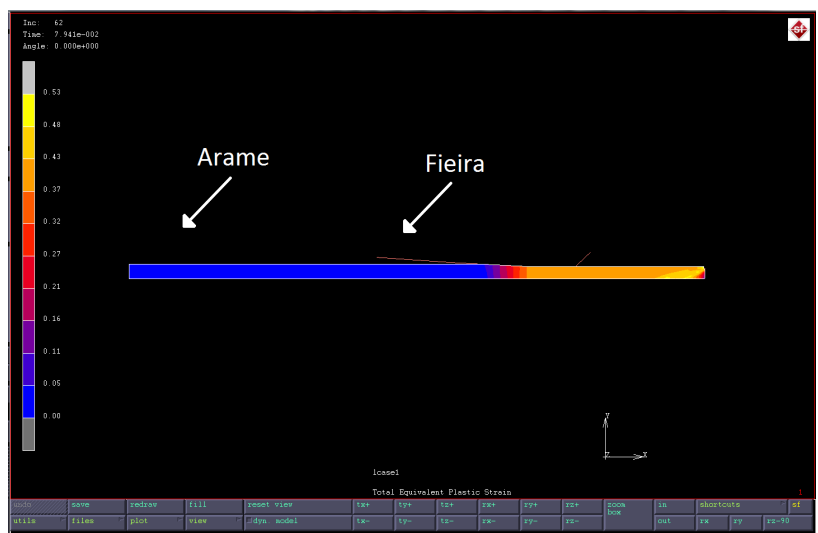

Figura 5. Momento em que o arame está sofrendo redução de área na simulação do processo de trefilação.

computacionais obteve-se um valor de $8376 \mathrm{~N}$ para a força de trefilação. Este valor implica em uma diferença de 35\% em relação à corrida I e $25 \%$ em relação à corrida II do material em estudo. 
Estas diferenças são consideradas altas, embora dentro do valor teórico aceitável para uma comparação entre experimento e simulações computacionais que é de $30 \%$, segundo critérios adotados pelo Instituto IUL de Dortmund Alemanha que é referência mundial em simulação. Este desvio deve-se a diversos fatores, dentre eles: consideração da curva real de escoamento do material, diferenças geométricas e erros de limitação do software, que utiliza a força máxima para fins de erros de projeto e dimensionamento.

\subsection{Deformação Plástica}

A Figura 6 demonstra um resultado global de deformação plástica de $41 \%$, valor que pode ser comprovado pela equação para a deformação real do processo. Este resultado é um indicativo de que o modelo numérico computacional foi eficiente para o cálculo das deformações.

$\mathrm{Na}$ Figura 7 são apresentados os resultados na malha da barra no software para a deformação plástica localizada para cada um dos elementos da barra após a trefilação.

Na Figura 8 são apresentados os resultados para a deformação plástica em cada um dos elementos do modelo desenvolvido. Como o modelo possuía cinco elementos radiais, foi calculada a média dos valores de deformação plástica para cinco elementos centrais, para que fossem evitados os efeitos do início e final da trefilação. Como foi desenvolvido um modelo axissimétrico, a média dos valores de deformação plástica para a metade da barra foi extrapolada para a barra toda, onde 0 representa o centro e -2,25 mm e 2,25 mm as extremidades.

Através da Figura 8 é possível perceber que os elementos do material, após a trefilação, sofrem deformação heterogênea. Para o centro da barra, o valor da deformação plástica atinge valores próximos a 0,4 (40\%), já para as regiões superficiais, a deformação plástica alcança valores em torno de 0,4I4 (4I,4\%).

Estes resultados demonstram que os elementos próximos à fieira sofreram maior deformação plástica que os elementos centrais. Esta deformação heterogênea do material sugere que o material irá apresentar um campo de tensões residuais após a realização do processo. Esta deformação heterogênea ocorre, pois o material está sofrendo tensões de tração e compressão de formas diferenciadas, sendo que algumas regiões como a central estão sofrendo tensões trativas, já as regiões superficiais estão sofrendo tensões cisalhantes.

\subsection{Tensões Residuais}

As Figuras 9, 10, I I e 12 apresentam o comparativo dos resultados de tensões residuais para as direções axial e radial simulados para o modelo de elementos finitos axissimétrico mecânico com fieira rígida e seus modelos computacionais desenvolvidos.

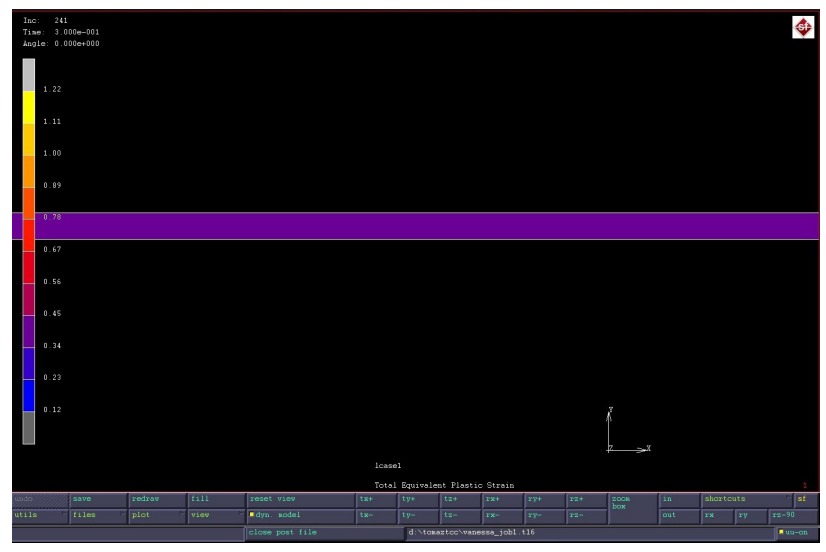

Figura 6. Resultado da deformação plástica global.

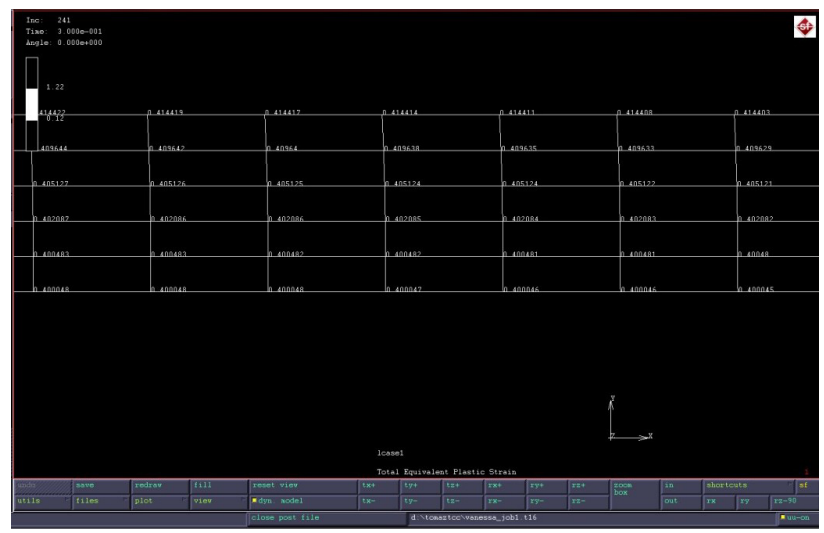

Figura 7. Resultados para a deformação plástica localizada.

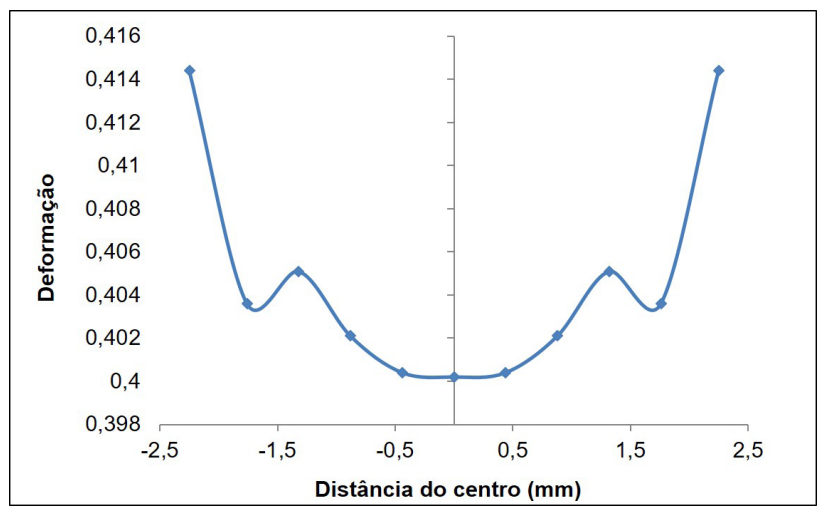

Figura 8. Resultados para a deformação plástica.

Como esperado na direção axial, para o centro da barra, as tensões apresentam comportamento compressivo; já para a superfície, apresentam comportamento trativo [II]. $\mathrm{Na}$ direção radial, as tensões são compressivas em toda a barra aproximando-se de zero na superfície. Esse comportamento dos perfis de tensões residuais é obtido porque a redução de área é maior que $1 \%$, caso contrário o sentido das tensões seria invertido.

Para a direção axial: Nas Figuras 10 e I I observa-se tensões trativas na superfície da barra com valores que 


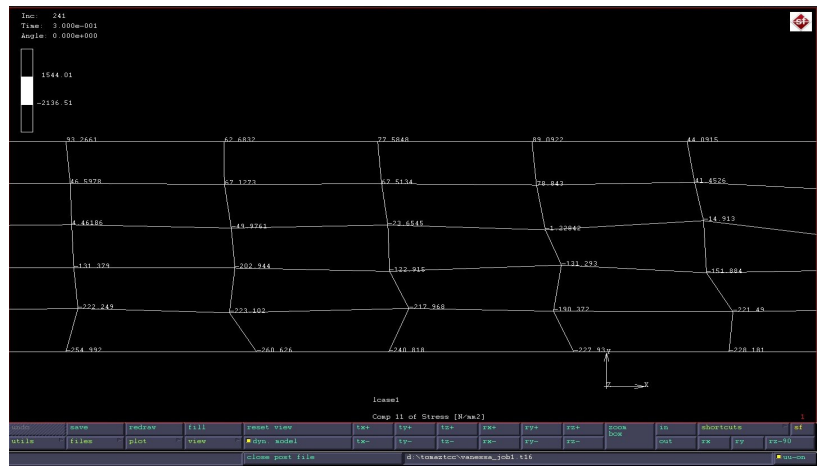

Figura 9. Tensões residuais axiais.

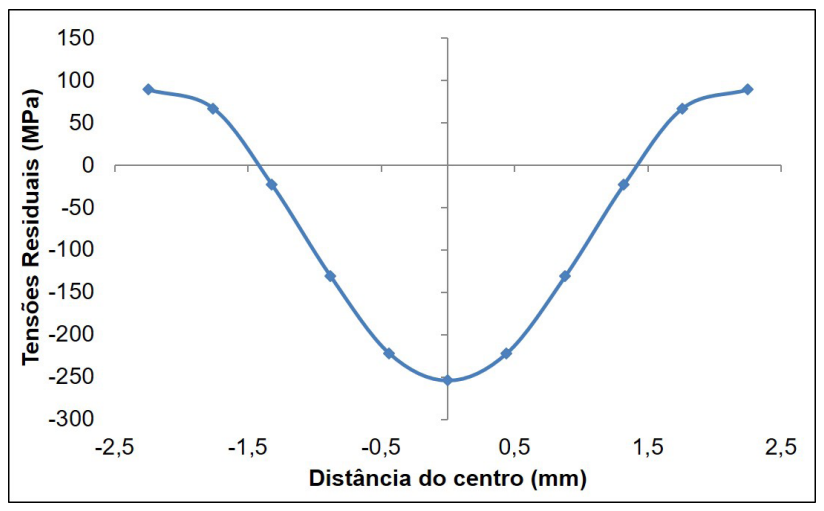

Figura 10. Gráfico tensões residuais axiais.

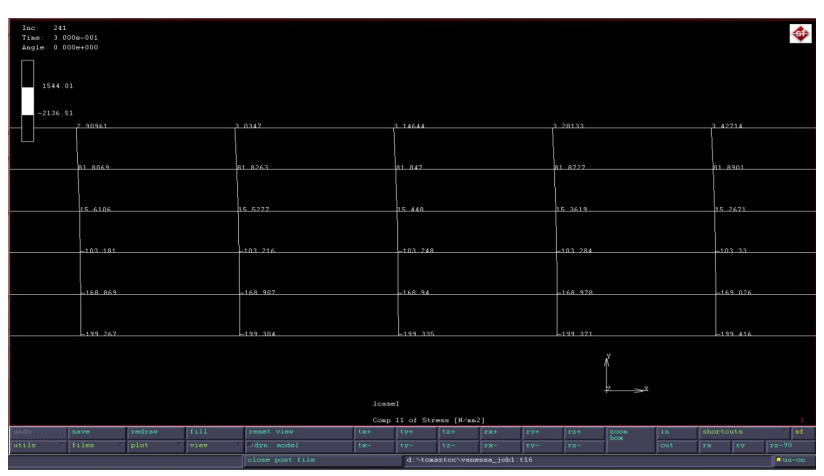

Figura I I. Tensões residuais radiais.

giram em torno de $89 \mathrm{MPa}$. No centro da barra, os valores das tensões calculadas aproximam-se de $-250 \mathrm{MPa}$ compressivos.

Para a direção radial: Na Figura 10 e Figura II a simulação apresenta resultado próximo a zero na superfície da barra e comportamento compressivo para o centro. $\mathrm{Na}$ região central o modelo apresentou um valor compressivo de - 199 Mpa.

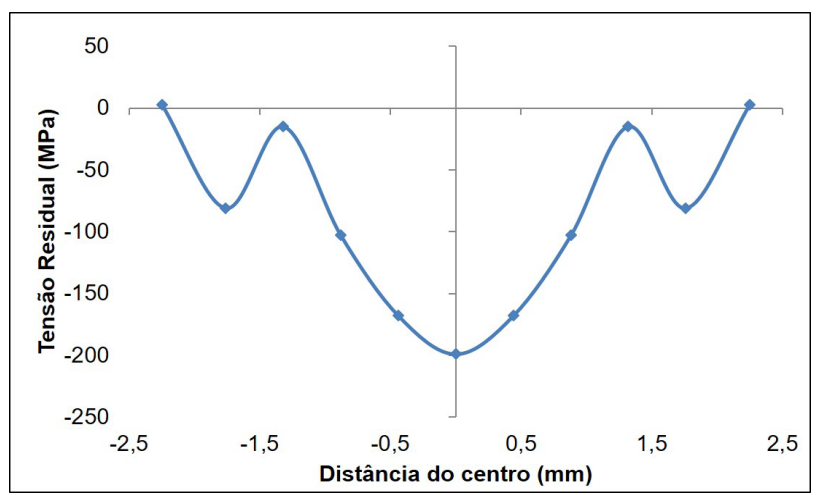

Figura 12. Gráfico tensões residuais radiais.

Analisando os perfis de tensões residuais pode se afirmar que as tensões envolvidas são de uma alta magnitude quando comparadas com a tensão de escoamento inicial do processo. Além disso, nota-se que os valores de tensões residuais para as direções axial e radial apresentam resultados próximos para o centro da barra devido às direções serem iguais nesse ponto, o que é consistente com a literatura pesquisada e as condições iniciais de modelamento $[12,13]$.

\section{CONCLUSÃO}

Ao longo deste trabalho foram discutidos diversos aspectos relativos ao processo da trefilação de arames de aço de baixo teor de carbono objetivando-se encontrar valores de força, deformações e tensões residuais. A partir dos resultados obtidos conclui-se que:

- Foi possível validar o modelo computacional simulado através da trefilação experimental do material em questão e da comparação da equação da força de Siebel;

- Foi possível reproduzir-se via elementos finitos o processo experimental da etapa de trefilação através de uma modelagem mecânica axissimétrica com fieira rígida. A validação da simulação foi realizada através da comparação da deformação global equivalente e dos perfis de tensões residuais obtidos;

- O modelo computacional de trefilação validado neste trabalho poderá futuramente ser aplicado em indústrias de trefilação para utilizar parâmetros ótimos no processo que agreguem maior eficiência energética, produtividade e redução de perda metálica, tornando assim o processo de trefilação mais sustentável tanto ambientalmente quanto economicamente. 


\section{REFERÊNCIAS}

I Cetlin PR. Trefilação básica. Belo Horizonte: Departamento de Engenharia Metalúrgica e de Materiais da UFMG; 2012.

2 Baid C, Wright RN. Flow stress for the modeling of stainless steel and nickel wire drawing. Wire Journal International. 20II; March: 66-69.

3 Lange K. Forming handbook. New York: McGraw Hill and SME; 1993.

4 Dieter GE. Metalurgia mecânica. 4. ed. Rio de Janeiro: Guanabara Dois; 1996.

5 Martins P, Rodrigues J. Tecnologia mecânica. vol. I. Lisboa: Escolar; 2005.

$6 \mathrm{Kim}$ T, Kim B, Choi J. Predictions of die wear in the wire-drawing process. Journal of Materials Processing Technology. 1997;65: I I-I7.

7 Nakagiri A, Yamano T, Konaka M, Asakawa M, Sasaki W. Behavior of residual stress and drawing stress in conicaltype die and circle-type die drawing by fem simulation and experiment. Wire Journal International. 2002; August: $72-80$

8 Schaeffer L. Conformação mecânica. Porto Alegre: Imprensa Livre; 2004.

9 Tekkaya AE. State-of-the-art of simulation of sheet metal forming. Journal of Materials Processing Technology. 2002; 103:14-22.

10 Godfrey H, Richards F, Saron S. The benefits of using wiredrawing dies with smaller included angles and longer nibs. Wire Journal International. 2000;33(6): 102-113.

II Martins P, Rodrigues J. Tecnologia mecânica. vol. I. Lisboa: Escolar; 2005.

12 Dieter GE. Metalurgia mecânica. 4. ed. Rio de Janeiro: Guanabara Dois; 1996.

13 Atienza JM, Elices M. Influence of residual stresses in the stress relaxation of cold drawn wires. Materials and Structures. 2004;37(269):30I-304.

Recebido em: 31 Ago. 2016

Aceito em: 10 Jan. 2017 